\title{
AST to platelet ratio index for predicting the in-hospital mortality in chronic liver disease patients in tertiary care hospital
}

\author{
Nagaraja BS ${ }^{1}$, Madhumathi $\mathbf{R}^{2}$, Avinash $\mathrm{HR}^{3}$, Umesh $\mathrm{KJ}^{4}$ \\ ${ }^{1}$ Professor and Head, ${ }^{2}$ Professor, ${ }^{3,4}$ Post graduate, Department of Medicine, Bangalore Medical College and Research \\ Institute, KR road, Bangalore, Karnataka, India
}

Background: Liver cirrhosis is the end result of chronic liver injury. Cirrhosis of liver may progressively deteriorate from a well-compensated state to decompensated conditions. Aims and Objective: Our study aims at evaluating the AST to Platelet Ratio Index (APRI) for predicting the in-hospital mortality and also comparing APRI, MELD and albumin for predicting in hospital mortality in chronic liver disease. Materials and Methods: Data of Patients with Chronic liver disease were retrospectively reviewed. MELD and APRI scores were calculated for the patients and results from ROC curves were analysed. Results: In our study conducted on 299 patients, the age distribution was between 18-64 years with mean age of patients being $46.47+/-10.9$ years, sex ratio Male: Female: $266: 37$ with mortality rate of $17.7 \%$. The Area under curves of ROC of APRI, MELD and Albumin are 0.63, 0.76 and 0.55 . Conclusion: APRI is an independent predictor of mortality. The prognostic performance of all 3 was comparable but MELD has better prognostic significance than APRI score.
Access this article online

\section{Website:}

http://nepjol.info/index.php/AJMS

DOI: 10.3126/ajms.v10i4.23170

E-ISSN: 2091-0576

P-ISSN: 2467-9100

Key words: Aspartate aminotransferase to platelet ratio index; Model of end stage liver disease; Area under curve; Alanine transaminase; Hepatitis $C$ virus

\section{INTRODUCTION}

Liver cirrhosis is the end result of fibrogenesis that occur with chronic liver injury and tissue repair due to any etiology. ${ }^{1}$ Alcohol is one of the common aetiology, alcohol related liver disease is the commonest cause of death, accounting for 2.5 million/yr. ${ }^{2}$ A cirrhotic liver may progressively deteriorate from compensated state to decompensated conditions. 5-year survival rate of $84 \%$ in compensated cirrhosis, $14 \%$ to $35 \%$ decompensated cirrhosis. $^{3-5}$ Although a preferable treatment option for decompensated liver cirrhosis is liver transplantation, shortage of donor livers and high cost make this approach impracticable in most cases. ${ }^{5-6}$ Therefore, identifying a marker associated with disease severity helps in improving clinical management. Aspartate aminotransferase to platelet ratio index (APRI) was initially proposed as a predictive marker for liver fibrosis and cirrhosis in hepatitis $C$ virus (HCV)-infected patients. ${ }^{7}$ Recent studies show that APRI predicted liver related mortality in alcoholic liver disease individuals. $^{8}$

APRI was calculated using the formula $=$ AST $(\mathrm{U} / \mathrm{L}) /$ (upper limit of the normal range) $\times 100 /$ platelet count $\left(10^{9} / \mathrm{L}\right)$. The $40 \mathrm{U} / \mathrm{L}$ of AST was used as the upper limit of the normal range. It is a mathematical formula using only two parameters, based on routine blood tests and is compared to MELD score and albumin level. APRI reflect extent of liver injury and compensatory state of hepatic function which is simpler, cost effective and easier to calculate than MELD score.

Herein we conducted a retrospective data analysis to determine APRI, the association of APRI with the progression of chronic alcoholic liver disease and mortality and to compare the prognostic significance of the APRI score with MELD score and albumin level. 


\section{AIMS AND OBJECTIVES}

1) To evaluate the AST to Platelet Ratio Index (APRI) for predicting the in-hospital mortality in chronic liver disease patients.

2) To compare APRI, MELD and albumin for predicting hospital mortality in chronic liver disease.

\section{MATERIALS AND METHODS}

The study was conducted at Bowring and Lady Curzon Hospital (Attached to Bangalore Medical College and Research institute). Chronic liver disease patients secondary to ethanol between 2016 January and 2016 December were retrospectively reviewed.

\section{Inclusion criteria}

1) All patients aged above 18 years with chronic liver disease.

2) Chronic liver disease as evidenced by abdominal ultra sound and liver profile derangement.

\section{Exclusion criteria}

1) Chronic liver disease due to hepatitis B, C, malignancy, metabolic causes, autoimmune hepatitis.

\section{Method of collection of data}

History, clinical Examination and Routine CBP, RFT, LFT, serum electrolytes, HIV, HBsAg, HCV, VDRL serology, prothrombin time, APT'T, Ultrasound of abdomen, upper GI endoscopy and other relevant investigations were noted.

Chronic liver disease is evidenced by: abdominal ultrasound and liver profile derangement.

Complications like anaemia, hepatic encephalopathy, renal dysfunction and mortality noted.

$\mathrm{APRI}=\mathrm{AST}(\mathrm{U} / \mathrm{L}) /($ upper limit of the normal range $)$ $\times 100 /$ platelet count.

The $40 \mathrm{U} / \mathrm{L}$ of AST was used as the upper limit of the normal range.

$\operatorname{MELD}=(10 *((0.957 * \ln ($ Creatinine $))+(0.378 * \ln$ $($ Bilirubin $))+(1.12 * \ln (\mathrm{INR}))+6.43$

Method of Statistical analysis

All statistical analysis were performed using the Medcalc software. Continuous Data were expressed as the mean+/- standard deviation (SD) and median with minimum and maximum. Categorical data were expressed as the frequency. Receiving-operative characteristics curve analysis was performed to identify the discriminative ability of the APRI, MELD score and albumin levels in predicting in-hospital mortality. Areas under the ROC curves were calculated and compared. The best cut off value was selected as the sum of sensitivity and specificity was maximal. Then sensitivity, specificity, positive likelihood ratio, negative likelihood ratio were reported.

\section{RESULTS AND ANALYSIS}

The sample size in our study was 299 patients. The age distribution was between 18-64 years with mean age of patients being $46.47+/-10.9$ years (Table 1$) .267$ were males and 32 were females (Table 2).

Among 299 patients, 53 were deaths and 246 were patients who showed improvement, with mortality percentage of $17.7 \%$ (Figure 1).

\begin{tabular}{lcc}
\multicolumn{3}{l}{ Table 1: Age distribution of patients studied } \\
\hline Age in years & No. of patients & $\%$ \\
\hline $20-30$ & 20 & 6.0 \\
$31-40$ & 68 & 22.7 \\
$41-50$ & 117 & 59.1 \\
$51-60$ & 63 & 21.0 \\
$61-70$ & 26 & 8.6 \\
$>70$ & 5 & 1.6 \\
Total & 299 & 100.0 \\
\hline Mean \pm SD: $46.47 \pm 10.9$ & &
\end{tabular}

\begin{tabular}{lcc} 
Table 2: Gender distribution of patients studied \\
\hline Gender & No. of patients & $\%$ \\
\hline Female & 32 & 10.7 \\
Male & 267 & 89.2 \\
Total & 299 & 100.0 \\
\hline *Death: 53, Improved: 246 & &
\end{tabular}

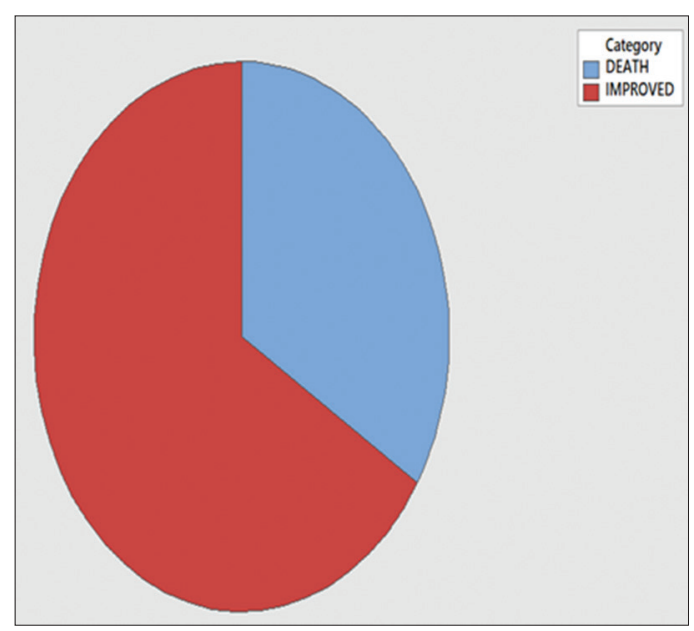

Figure 1: Outcome 
Comparison of in-Hospital mortality with APRI, MELD and albumin level

The in-Hospital morality was $17.7 \%$. The Area under curve (AUC) of the APRI score for predicting the in-hospital mortality was 0.631 (confidence interval: 95\%: $0.574-0.686$ ) (Table 3). The best cut-off value of -0.743 , with sensitivity of $77.9 \%$, a specificity of $46.2 \%$, positive likelihood ratio (PLR) of 1.72 and negative likelihood ratio (NLR) 1.66 (Figure 2).

The AUC of the MELD score for predicting the in-hospital mortality was 0.766 (confidence interval 95\%: 0.713-0.812), with a sensitivity of $84.2 \%$, a specificity of $75.6 \%$, PLR of 1.7 and NLR of 1.6 (Figure 3).

The AUC of the Albumin level for predicting the inhospital mortality was 0.559 (confidence interval 95\% $0.500-0.616$ ), with a sensitivity of $89.8 \%$, a specificity of $28.7 \%$, PLR of 3.23 and NLR of 3.23 (Figure 4).

The AUC for predicting the in-hospital mortality was significantly different between the APRI, MELD score

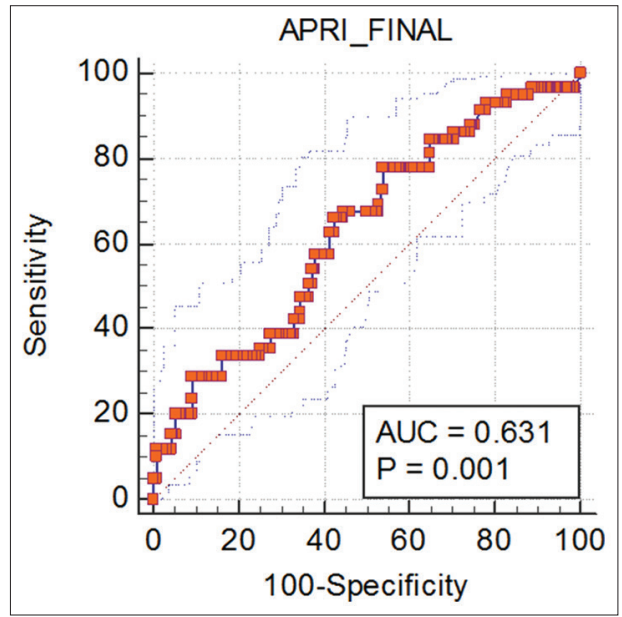

Figure 2: Area under curve for APRI

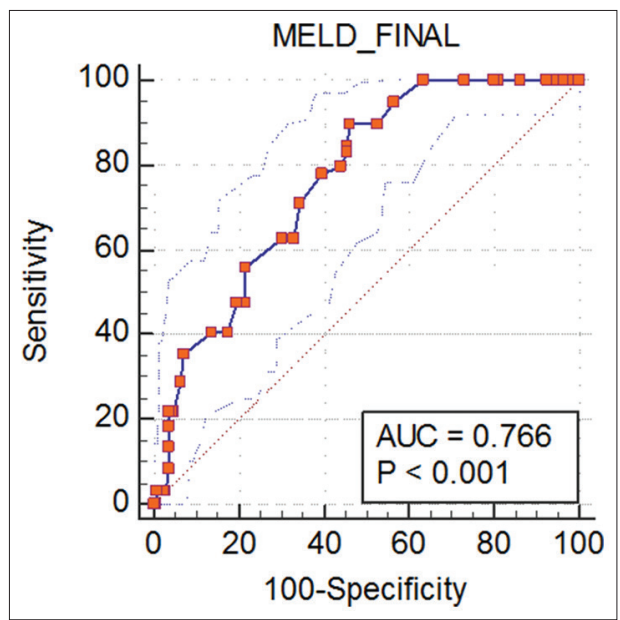

Figure 3: Area under curve for MELD score and albumin level (Figure 5). (APRI and MELD score $-\mathrm{P}=0.0015$; Albumin level and MELD $\mathrm{P}<0.001)$ (Table 4).

ORDER: MELD score > APRI > Albumin level.

\section{DISCUSSION}

Cirrhosis of liver is associated with significant morbidity, mortality and health care costs. There are several biochemical markers that can reveal both liver function and the extent of liver injury. ${ }^{9}$ APRI uses platelet count and AST levels to reflect insufficient liver function and new stress/damage to the liver. Decreased platelet

\begin{tabular}{lccc}
\multicolumn{4}{l}{ Table 3: Area under curve } \\
\hline Variable & AUC & Standard error & $\mathbf{9 5 \%} \mathbf{~ C l}$ \\
\hline ALBUMIN & 0.559 & 0.0392 & 0.500 to 0.616 \\
APRI_FINAL & 0.631 & 0.0403 & 0.574 to 0.686 \\
MELD_FINAL & 0.766 & 0.0300 & 0.713 to 0.812 \\
\hline
\end{tabular}

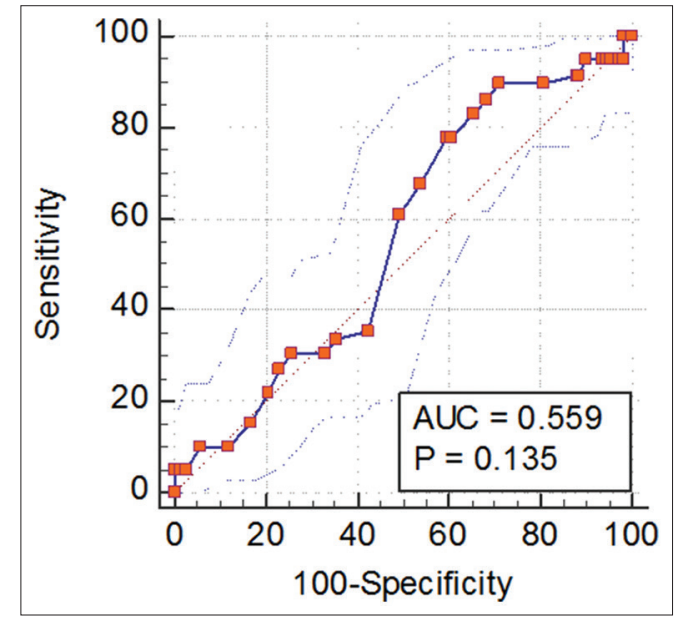

Figure 4: Area under curve for Albumin

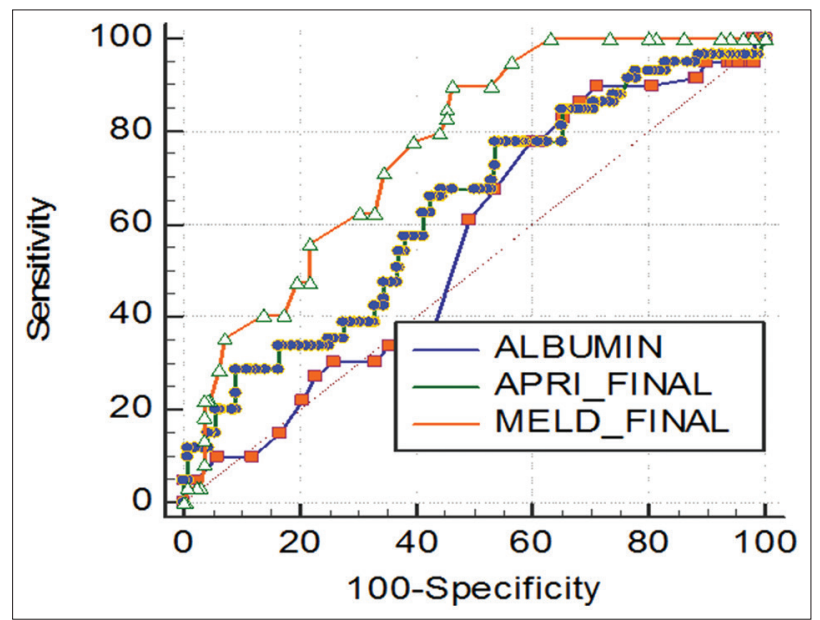

Figure 5: comparing AUC for albumin, MELD and APRI

Asian Journal of Medical Sciences | Jul-Aug 2019 | Vol 10 | Issue 4 


\begin{tabular}{lc}
\multicolumn{2}{l}{ Table 4: Pairwise comparison of ROC curves } \\
\hline \multicolumn{2}{l}{ ALBUMIN APRI_FINAL } \\
\hline Difference between areas & 0.0730 \\
Standard Error & \\
95\% Confidence Interval & 0.0557 \\
z statistic & -0.0361 to 0.182 \\
Significance level & 1.311 \\
\hline ALBUMIN MELD_FINAL & $\mathrm{P}=0.1899$ \\
\hline Difference between areas & \\
Standard Error & \\
95\% Confidence Interval & 0.207 \\
z statistic & 0.0461 \\
Significance level & 0.117 to 0.297 \\
\hline APRI_FINAL MELD_FINAL & 4.491 \\
\hline Difference between areas & $\mathrm{P}<0.0001$ \\
Standard Errora & \\
95\% Confidence Interval & 0.134 \\
z statistic & 0.0423 \\
Significance level & 0.0512 to 0.217 \\
\hline
\end{tabular}

count and increased AST levels are known as clinical manifestations of progression of liver cirrhosis. A reduction in platelet count can be caused by the accelerating destruction of an enlarged spleen which is termed as "hypersplenism" secondary to portal hypertension in cirrhosis. ${ }^{10-12}$ AST is more abundantly present in the mitochondria and cytoplasm relative to ALT. ${ }^{13}$ Fibrosis of liver may reduce the clearance of AST, leading to the retention of AST in blood. ${ }^{13-15}$ Therefore, high AST levels combined with low platelet count may be used to predict the severity and progression of liver injury in cirrhotic patients.

In our study, we tried to explore the prognostic performance of the APRI score for the assessment of the in-hospital mortality of chronic liver disease.

We found that the prognostic performance of the APRI score was comparable to that of MELD score and albumin level. In this study, MELD score had the largest AUC, followed by APRI and albumin level. Therefore, MELD score has better prognostic performance compared to APRI and albumin level.

Study done by Weilin Mao et al, On 193 chronic HBVinfected patients. Mortality that occurred within 90 days of hospital stay was compared, which concludes that APRI is an independent predictor for mortality in patients with cirrhosis and they found positive correlation between the MELD score and APRI.?

In a study conducted by Lieber CS1 et al, on 1308 patients, APRI has low sensitivity and specificity for the diagnosis of significant fibrosis in patients with alcoholic liver disease. ${ }^{8}$

\section{Limitation of the study}

1) Long term follow up was unavailable so this study couldn't evaluate the role of APRI for predicting long term Prognosis.

2) APRI score is not dynamically measured.

3) Retrospective study, needs prospective study for better understanding of correlation.

\section{CONCLUSION}

APRI is an independent predictor of mortality. Highly elevated APRI was associated with higher frequencies of clinical complications such as ascites and encephalopathy

The prognostic performance of all 3 variables were comparable but MELD score has better prognostic significance than APRI score

\section{REFERENCES}

1. Dooley J, Lok A, Garcia-Tsao G, Pinzani M. Sherlock's diseases of the liver and biliary system. $12^{\text {th }}$ ed. UK. Wiley-black well professional publishing; 6, 103-118 (2007).

2. Mark E Mailliard, Micheal F Sorell. Alcoholic Liver Disease. Harrison's Principles of Internal Medicine. $19^{\text {th }}$ ed. New York. McGraw Hill; 2015: p2052.

3. Tsochatzis EA, Bosch $\mathrm{J}$ and Burroughs AK. Liver cirrhosis. Lancet.2014; 383:1749-1761.

4. Kamath PS and Kim WR. Advanced Liver Disease Study group, The Model for end stage liver disease (MELD). Hepatology 2007; 45(3):797-805.

5. Chung GE, Lee JH and Kim YJ. Does antiviral therapy reduce complications of cirrhosis. World J Gastroenterol 2014; 20(23):7306-7311.

6. Albert C, Fan ST, Lo CM, Liu CL, Chan SC, Kelvin K, et al, Liver transplantation for acute-on chronic liver failure. Hepatol Int 2009; 3:571-581.

7. Wai CT, Greenson JK, Fontana RJ, Kalbfleisch JD, Marrero JA, Conjeevaram HS, et al, A simple non-invasive index can predict both significant fibrosis and cirrhosis in patients with chronic hepatitis C. Hepatology 2003; 38(2):518-526.

8. Lieber CS, Weiss DG and Morgan TR. Aspartate aminotransferase to platelet ratio index in patients with alcoholic liver fibrosis. Am J Gastroenterol 2006; 101: 1500-1508.

9. Mao W, Sun Q, Fan J, Lin S and Ye B. AST to Platelet Ratio Index Predicts Mortality in Hospitalized Patients With Hepatitis B-Related Decompensated Cirrhosis. Medicine 2016; 95(9):e2946.

10. Toghill PJ, Green S and Ferguson F. Platelet dynamics in chronic liver disease with special reference to the role of the spleen. J Clin Pathol 1977; 30:367-371.

11. Aster RH. Pooling of platelets in the spleen: role in the pathogenesis of "hypersplenic" thrombocytopenia. J Clin Invest 1966; 45:645-657.

12. McCormick PA and Murphy KM. Splenomegaly, hypersplenism and coagulation abnormalities in liver disease. Baillieres Best Pract Res Clin Gastroenterol 2000; 14:1009-1031. 
13. Okuda M, Kui Li, Beard MR, Lori A, Showalter, Scholle F, et al, Mitochondrial injury, oxidative stress, and antioxidant gene expression are induced by hepatitis $\mathrm{C}$ virus core protein. Gastroenterology 2002; 122:366-375.

14. Nalpas B, Vassault A, Le Guillou A, Lesgourgues B, Ferry N, Lacour B, et al. Serum activity of mitochondrial aspartate aminotransferase: a sensitive marker of alcoholism with or without alcoholic hepatitis. Hepatology 1984; 4(5):893-896.

15. Kamimoto $\mathrm{Y}$, Horiuchi $\mathrm{S}$, Tanase $\mathrm{S}$ and Morino $\mathrm{Y}$, Plasma clearance of intravenously injected aspartate aminotransferase isozymes: evidence for preferential uptake by sinusoidal liver cells. Hepatology1985; 5(3):367-375.

\section{Authors Contribution:}

NBS- Concept and Design of the study, Manuscript preparation, Statistically analysed and interpreted, critical revision of the manuscript; MR- Concept and Design of the study, Statistically analysed and interpreted, critical revision of the manuscript; AHR- reviewed the literature, helped in preparing first draft of Manuscript, collected data; UKJ - collected data, Statistically analysed and interpreted, helped in preparing draft of manuscript.

Work attributed to:

Department of General Medicine, Hospitals attached to Bangalore Medical College and Research institute.

\section{Orcid ID:}

Dr. Nagaraja BS- (iD https://orcid.org/0000-0002-7750-697X

Dr. Madhumathi R- (iD https://orcid.org/0000-0002-7534-1059

Dr. Avinash HR- (1) https://orcid.org/0000-0001-6484-5190

Dr. Umesh KJ- (1) https://orcid.org/0000-0002-1816-9125

Source of Support: Bangalore Medical College and Research Institute fund, Conflict of Interest: None. 